\title{
INEQUALITIES OF OPIAL TYPE IN THREE INDEPENDENT VARIABLES
}

\author{
B. G. PACHPATTE
}

\begin{abstract}
The aim of the present paper is to establish some new integral inequalities of Opial type involving functions of three independent variables and their partial derivatives. Our results yields in the special cases some of the inequalities recently appeared in the literature.
\end{abstract}

\section{Introduction}

In 1960, Z. Opial [4] established the following inequality.

Theorem A. If $f \in C^{1}[o, h]$ satisfies $f(o)=f(h)=0$ and $f(x)>0$ for $x \in(o, h)$, then

$$
\int_{o}^{h}\left|f(x) f^{\prime}(x)\right| d x \leq \frac{h}{4} \int_{o}^{h} f^{\prime}(x)^{2} d x
$$

where the constant factor $\frac{h}{4}$ is best possible.

The inequality (1.1) has received a wide attention in the literature, and in numerous papers new proofs, extensions, variants, and discrete analogues of inequality (1.1) are presented, see [1-12] and the references cited therein. In a recent paper [11], Salem obtained the following analogue of (1.1) in three independent variables.

Theorem B. Let $f(r, s, t), f_{1}(r, s, t), f_{12}(r, s, t)$ and $f_{123}(r, s, t)$ be continuous functions on $\triangle=[a, k] \times[b, m] \times[c, n]$ and let $f(a, s, t)=f(k, s, t)=f(r, b, t)=f(r, m, t)=$ $f(r, s, c)=f(r, s, n)=0$ for $a \leq r \leq k, b \leq s \leq m, c \leq t \leq n$. Then

$$
\begin{aligned}
& \int_{a}^{k}\left(\int_{b}^{m}\left(\int_{c}^{n}|f(r, s, t)|\left|f_{123}(r, s, t)\right| d t\right) d s\right) d r \\
\leq & M \int_{a}^{k}\left(\int_{b}^{m}\left(\int_{c}^{n}\left|f_{123}(r, s, t)\right|^{2} d t\right) d s\right) d r,
\end{aligned}
$$

where

$$
M=\frac{(k-a)(m-b)(n-c)}{16},
$$

Received October 2, 2002

2000 Mathematics Subject Classification. 26D10, 26D15.

Key words and phrases. Inequalities of opial type, three independent variables, Schwarz inequality, Hölder's inequality. 
and $f_{1}(r, s, t)=\frac{\partial}{\partial r} f(r, s, t), f_{12}(r, s, t)=\frac{\partial^{2}}{\partial r \partial s} f(r, s, t), f_{123}(r, s, t)=\frac{\partial^{3}}{\partial r \partial s \partial t} f(r, s, t)$ for $(r, s, t) \in \triangle$.

The inequality (1.2) is motivated by the similar inequality in two independent variables established by Yang in [12], see also [5-10]. The main object of this paper is to establish some new integral inequalities which are the three independent variables analogues of some of the inequalities recently established by Pachpatte in [5-10]. An interesting feature of our results is that, the analysis used in their proofs is quite elementary and in the special cases they yield the inequality in (1.2) as well as some other inequalities given in [11, Theorem 2].

\section{Statement of Results}

In what follows we denote by $R$ the set of real numbers. For $a \leq x \leq k, b \leq y \leq m, c \leq$ $z \leq n$, where $a, x, k, b, y, m, c, z, n$ in $R$, we define $\triangle=[a, k] \times[b, m] \times[c, n], \triangle_{1}=[a, x] \times$ $[b, y] \times[c, z], \triangle_{2}=[a, x] \times[b, y] \times[z, n], \triangle_{3}=[a, x] \times[y, m] \times[c, z], \triangle_{4}=[x, k] \times[b, y] \times[c, z]$, $\triangle_{5}=[a, x] \times[y, m] \times[z, n], \triangle_{6}=[x, k] \times[y, m] \times[c, z], \triangle_{7}=[x, k] \times[b, y] \times[z, n]$, $\triangle_{8}=[x, k] \times[y, m] \times[z, n]$. If $f(r, s, t)$ is a differentiable function defined on $\triangle$, then its partial derivatives are denoted by $D_{1} f(r, s, t)=\frac{\partial}{\partial r} f(r, s, t), D_{2} f(r, s, t)=\frac{\partial}{\partial s} f(r, s, t)$, $D_{3} f(r, s, t)=\frac{\partial}{\partial t} f(r, s, t), D_{2} D_{1} f(r, s, t)=\frac{\partial^{2}}{\partial s \partial r} f(r, s, t), D_{3} D_{2} f(r, s, t)=\frac{\partial^{2}}{\partial t \partial s} f(r, s, t)$, $D_{3} D_{1} f(r, s, t)=\frac{\partial^{2}}{\partial t \partial r} f(r, s, t), D_{3} D_{2} D_{1} f(r, s, t)=\frac{\partial^{3}}{\partial t \partial s \partial r} f(r, s, t)$. For $a \leq x \leq k$, $b \leq y \leq m, c \leq z \leq n$ and for some suitable fucntions $f(x, y, z), I_{j} D_{3} D_{2} D_{1} f(x, y, z)$, $(j=1,2, \ldots, 8)$ defined on $\triangle$, we set

$$
\begin{array}{ll}
I_{1} D_{3} D_{2} D_{1} f=\int_{a}^{x} \int_{b}^{y} \int_{c}^{z}\left|D_{3} D_{2} D_{1} f(r, s, t)\right| d t d s d r, \quad(x, y, z) \in \triangle_{1}, \\
I_{2} D_{3} D_{2} D_{1} f=\int_{a}^{x} \int_{b}^{y} \int_{z}^{n}\left|D_{3} D_{2} D_{1} f(r, s, t)\right| d t d s d r, \quad(x, y, z) \in \triangle_{2}, \\
I_{3} D_{3} D_{2} D_{1} f=\int_{a}^{x} \int_{y}^{m} \int_{c}^{z}\left|D_{3} D_{2} D_{1} f(r, s, t)\right| d t d s d r, \quad(x, y, z) \in \triangle_{3}, \\
I_{4} D_{3} D_{2} D_{1} f=\int_{x}^{k} \int_{b}^{y} \int_{c}^{z}\left|D_{3} D_{2} D_{1} f(r, s, t)\right| d t d s d r, \quad(x, y, z) \in \triangle_{4}, \\
I_{5} D_{3} D_{2} D_{1} f=\int_{a}^{x} \int_{y}^{m} \int_{z}^{n}\left|D_{3} D_{2} D_{1} f(r, s, t)\right| d t d s d r, \quad(x, y, z) \in \triangle_{5}, \\
I_{6} D_{3} D_{2} D_{1} f=\int_{x}^{k} \int_{y}^{m} \int_{c}^{z}\left|D_{3} D_{2} D_{1} f(r, s, t)\right| d t d s d r, \quad(x, y, z) \in \triangle_{6}, \\
I_{7} D_{3} D_{2} D_{1} f=\int_{x}^{k} \int_{b}^{y} \int_{z}^{n}\left|D_{3} D_{2} D_{1} f(r, s, t)\right| d t d s d r, \quad(x, y, z) \in \triangle_{7}, \\
I_{8} D_{3} D_{2} D_{1} f=\int_{x}^{k} \int_{y}^{m} \int_{z}^{n}\left|D_{3} D_{2} D_{1} f(r, s, t)\right| d t d s d r, \quad(x, y, z) \in \triangle_{8},
\end{array}
$$


where we have used the notation $I_{j} D_{3} D_{2} D_{1} f$ for $I_{j} D_{3} D_{2} D_{1} f(x, y, z)$. For some suitable functions $f, D_{3} D_{2} D_{1} f, H, H^{\prime}$ and $f_{i}, D_{3} D_{2} D_{1} f_{i}, H_{i}, H_{i}^{\prime}, i=1,2, \ldots, N, N \geq 2$, defined on the respective domains of their definitions, we set

$$
L\left[f, D_{3} D_{2} D_{1} f, H, H^{\prime}\right]=\left[H(|f(r, s, t)|)+H^{\prime}(|f(r, s, t)|)|f(r, s, t)|\right] \times\left|D_{3} D_{2} D_{1} f(r, s, t)\right|,
$$

and

$$
\begin{aligned}
& M\left[H_{1}, \ldots, H_{N}, H_{1}^{\prime}, \ldots, H_{N}^{\prime}, D_{3} D_{2} D_{1} f_{1}, \ldots, D_{3} D_{2} D_{1} f_{N}\right] \\
= & H_{1} \cdots H_{N-1} H_{N}^{\prime}\left|D_{3} D_{2} D_{1} f_{N}\right|+H_{1} \cdots H_{N-2} H_{N-1}^{\prime}\left|D_{3} D_{2} D_{1} f_{N-1}\right| H_{N} \\
& +\cdots+H_{1}^{\prime}\left|D_{3} D_{2} D_{1} f_{1}\right| H_{2} \cdots H_{N},
\end{aligned}
$$

where' dentoes the derivative of a function, and we have written $H$ for $H(|f(r, s, t)|)$, $H^{\prime}$ for $H^{\prime}(|f(r, s, t)|), D_{3} D_{2} D_{1} f$ for $D_{3} D_{2} D_{1} f(r, s, t)$ and $H_{i}$ for $H_{i}\left(\left|f_{i}(r, s, t)\right|\right), H_{i}^{\prime}$ for $H_{i}^{\prime}\left(\left|f_{i}(r, s, t)\right|\right), D_{3} D_{2} D_{1} f_{i}$ for $D_{3} D_{2} D_{1} f_{i}(r, s, t), i=1,2, \ldots, N$.

For convenience we list the following hypotheses.

$\left(\mathrm{A}_{1}\right)$ Let $f(r, s, t), D_{1} f(r, s, t), D_{2} D_{1} f(r, s, t)$ and $D_{3} D_{2} D_{1} f(r, s, t)$ be real-valued continuous functions defined on $\triangle$ and $f(a, s, t)=f(k, s, t)=f(r, b, t)=$ $f(r, m, t)=f(r, s, c)=f(r, s, n)=D_{1} f(r, b, t)=D_{1} f(r, m, t)=D_{2} D_{1} f(r, s, c)=$ $D_{2} D_{1} f(r, s, n)=0$ for $a \leq r \leq k, b \leq s \leq m, c \leq t \leq n$.

$\left(\mathrm{A}_{2}\right)$ Let $H(p)$ be a real-valued continuous function defined for all $p$ in the range of the function $f(r, s, t)$ and for all $p(r, s, t)$ of the forms $\int_{a}^{r} \int_{b}^{s} \int_{c}^{t}\left|D_{3} D_{2} D_{1} f(u, v, w)\right| d w d v d u$ for $(r, s, t) \in \triangle_{1}$ and for similar integrals for $(r, s, t)$ on $\triangle_{i}, i=2,3, \ldots, 8,|H(p)| \leq H(|p|)$ for all $p$ and that $H\left(p_{1}\right) \leq H\left(p_{2}\right)$ for $0 \leq p_{1} \leq p_{2}$.

$\left(\mathrm{A}_{3}\right)$ Let $H(p)$ be a convex increasing function for $p>0$ and $H(0)=0$.

$\left(\mathrm{A}_{4}\right)$ Let $H(p)$ be a nonnegative continuous nondecreasing real-valued function for $p \geq 0$ such that $H^{\prime}(p)$ exists, nonnegative, continuous and nondecreasing for $p \geq 0$.

$\left(\mathrm{A}_{5}\right)$ Let $f_{i}(r, s, t), D_{1} f_{i}(r, s, t), D_{2} D_{1} f_{i}(r, s, t), D_{3} D_{2} D_{1} f_{i}(r, s, t)$ be real-valued continuous functions defined on $\triangle$ and $f_{i}(a, s, t)=f_{i}(k, s, t)=f_{i}(r, b, t)=$ $f_{i}(r, m, t)=f_{i}(r, s, c)=f_{i}(r, s, n)=D_{1} f_{i}(r, b, t)=D_{1} f_{i}(r, m, t)=D_{2} D_{1} f_{i}(r, s, c)$ $=D_{2} D_{1} f_{i}(r, s, n)=0$ for $i=1,2, \ldots, N, N \geq 2$, and for $a \leq r \leq k, b \leq s \leq m$, $c \leq t \leq n$.

$\left(\mathrm{A}_{6}\right)$ Let $H_{i}(p)$ be real-valued, nonnegative, continuous, nondecreasing functions for $p \geq$ 0 and $H_{i}(0)=0$ such that $H_{i}^{\prime}(p)$ exist, nonnegative, continuous and nondecreasing for $p \geq 0$ and $i=1,2, \ldots, N, N \geq 2$.

Our main results are established in the following theorems.

Theorem 1. Assume that the hypotheses $\left(\mathrm{A}_{1}\right)$ and $\left(\mathrm{A}_{2}\right)$ hold. Then

$$
\int_{a}^{k} \int_{b}^{m} \int_{c}^{n}\left|H(f(r, s, t)) D_{3} D_{2} D_{1} f(r, s, t)\right| d t d s d r \leq \sum_{j=1}^{8} F\left(I_{j} D_{3} D_{2} D_{1} f\right),
$$

for $a \leq x \leq k, b \leq y \leq m, c \leq z \leq n$, where $F(p)=\int_{0}^{p} H(\sigma) d \sigma, p>0$. 
Remark 1. By taking $H(p)=p$ and hence $F(p)=\frac{p^{2}}{2}$ in Theorem 1, and applying Schwarz inequality to each integral on the right side of $(2.1)$ and then substituting $x=$ $\frac{a+k}{2}, y=\frac{b+m}{2}, z=\frac{c+n}{2}$, we get the inequality given in Theorem B. If we take $H(p)=p^{\alpha}$, where $\alpha \geq 0$ is a constant and hence $F(p)=\frac{p^{\alpha+1}}{\alpha+1}$ in Theorem 1, and apply Hölder's inequality with indices $\alpha+1,(\alpha+1) / \alpha$ to each integral on the right side of $(2.1)$, and take $x=\frac{a+k}{2}, y=\frac{b+m}{2}, z=\frac{c+n}{2}$, we get the following inequality

$$
\begin{aligned}
& \int_{a}^{k} \int_{b}^{m} \int_{c}^{n}|f(r, s, t)|^{\alpha}\left|D_{3} D_{2} D_{1} f(r, s, t)\right| d t d s d r \\
\leq & \frac{1}{\alpha+1}\left[\frac{(k-a)(m-b)(n-c)}{8}\right]^{\alpha} \int_{a}^{k} \int_{b}^{m} \int_{c}^{n}\left|D_{3} D_{2} D_{1} f(r, s, t)\right|^{\alpha+1} d t d s d r .
\end{aligned}
$$

we note that the inequality (2.2) is recently established by Salem in [11, Theorem 2].

Theorem 2. Assume that the hypotheses $\left(\mathrm{A}_{1}\right)$ and $\left(\mathrm{A}_{2}\right)$ hold. Then

$$
\int_{a}^{k} \int_{b}^{m} \int_{c}^{n}\left|\frac{D_{3} D_{2} D_{1} f(r, s, t)}{H(f(r, s, t))}\right| d t d s d r \geq \sum_{j=1}^{8} G\left(I_{j} D_{3} D_{2} D_{1} f\right),
$$

for $a \leq x \leq k, b \leq y \leq m, c \leq z \leq n$, where $G(p)=\int_{0}^{p} \frac{1}{H(\sigma)} d \sigma, p>0$.

Remark 2. We note that the inequality given in Theorems 1 and 2 can be considered as further extensions of the inequalities established by Pachpatte in [7] and we believe that these inequalities are of independent interest.

Theorem 3. Assume that the hypotheses $\left(\mathrm{A}_{1}\right)$ and $\left(\mathrm{A}_{3}\right)$ hold. Then

$$
\int_{a}^{k} \int_{b}^{m} \int_{c}^{n} H^{\prime}(|f(r, s, t)|)\left|D_{3} D_{2} D_{1} f(r, s, t)\right| d t d s d r \leq \sum_{j=1}^{8} H\left(I_{j} D_{3} D_{2} D_{1} f\right),
$$

for $a \leq x \leq k, b \leq y \leq m, c \leq z \leq n$.

Remark 3. We note that the inequalities established in Theorem 3 is motivated by the similar inequality established by Pachpatte in [7]. By taking $H(p)=p^{2}$ and hence $H^{\prime}(p)=2 p$ in (2.4) and applying Schwarz inequality to each integral on the right side of (2.4) and then substituting $x=\frac{a+k}{2}, y=\frac{b+m}{2}, z=\frac{c+n}{2}$, it is easy to observe that the inequlaity (2.4) reduces to the inequality given in Theorem B. If we take $H(p)=p^{\alpha+1}$, where $\alpha \geq 0$ is a constant, and hence $H^{\prime}(p)=(\alpha+1) p^{\alpha}$ in (2.4) and apply Hölder's inequality with indices $\alpha+1,(\alpha+1) / \alpha$ to each integral on the right side of $(2.4)$, and take $x=\frac{a+k}{2}, y=\frac{b+m}{2}, z=\frac{c+n}{2}$, we get the inequality obtained in (2.2).

Theorem 4. Assume that the hypotheses $\left(\mathrm{A}_{1}\right)$ and $\left(\mathrm{A}_{4}\right)$ hold. Then

$$
\int_{a}^{k} \int_{b}^{m} \int_{c}^{n} L\left[f, D_{3} D_{2} D_{1} f, H, H^{\prime}\right] d t d s d r \leq \sum_{j=1}^{8} H\left(I_{j} D_{3} D_{2} D_{1} f\right)\left(I_{j} D_{3} D_{2} D_{1} f\right)
$$


for $a \leq x \leq k, b \leq y \leq m, c \leq z \leq n$.

Remark 4. By letting $H(p)=p^{\alpha}$, where $\alpha \geq 1$ is a constant and hence $H^{\prime}(p)=$ $\alpha p^{\alpha-1}$ in (2.5) and applying Hölder's inequality with indices $\alpha+1,(\alpha+1) / \alpha$ to each integral on the right side, and then substituting $x=\frac{a+k}{2}, y=\frac{b+m}{2}, z=\frac{c+n}{2}$, we get the inequality given in (2.2). We note that the inequality (2.5) is motivated by the inequality recently established by Pachpatte in [10].

Theorem 5. Assume that the hypotheses $\left(\mathrm{A}_{5}\right)$ and $\left(\mathrm{A}_{6}\right)$ hold. Then

$$
\begin{aligned}
& \int_{a}^{k} \int_{b}^{m} \int_{c}^{n} M\left[H_{1}, \ldots, H_{N}, H_{1}^{\prime}, \ldots, H_{N}^{\prime}, D_{3} D_{2} D_{1} f_{1}, \ldots, D_{3} D_{2} D_{1} f_{N}\right] d t d s d r \\
\leq & \sum_{j=1}^{8}\left(\prod_{i=1}^{N} H_{i}\left(I_{j} D_{3} D_{2} D_{1} f_{i}\right)\right),
\end{aligned}
$$

for $a \leq x \leq k, b \leq y \leq m, c \leq z \leq n$.

Remark 5. We note that, the inequality given in Theorem 5 can be considered as a further extension of the inequality given by Pachpatte in [9]. In the special case when $f_{i}=f$ and $H_{i}=H$, then the inequality (2.6) reduces to

$$
\begin{aligned}
& \int_{a}^{k} \int_{b}^{m} \int_{c}^{n}\{H(|f(r, s, t)|)\}^{N-1} H^{\prime}(|f(r, s, t)|)\left|D_{3} D_{2} D_{1} f(r, s, t)\right| d t d s d r \\
\leq & \frac{1}{N} \sum_{j=1}^{8}\left\{H\left(I_{j} D_{3} D_{2} D_{1} f\right)\right\}^{N},
\end{aligned}
$$

for $a \leq x \leq k, b \leq y \leq m, c \leq z \leq n$. If we take $N=2$ in (2.7), then we get the following inequality

$$
\begin{aligned}
& \int_{a}^{k} \int_{b}^{m} \int_{c}^{n} H(|f(r, s, t)|) H^{\prime}(|f(r, s, t)|)\left|D_{3} D_{2} D_{1} f(r, s, t)\right| d t d s d r \\
\leq & \frac{1}{2} \sum_{j=1}^{8}\left\{H\left(I_{j} D_{3} D_{2} D_{1} f\right)\right\}^{2} .
\end{aligned}
$$

By taking $H(p)=p^{\alpha+1}$ in (2.8), where $\alpha \geq 0$ is a constant and hence $H^{\prime}(p)=(\alpha+1) p^{\alpha}$ and using the Hölder's inequality with indices $2(\alpha+1), 2(\alpha+1) /(2 \alpha+1)$ to the resulting integrals on the right side and then taking $x=\frac{a+k}{2}, y=\frac{b+m}{2}, z=\frac{c+n}{2}$, we get the following inequality

$$
\begin{aligned}
& \int_{a}^{k} \int_{b}^{m} \int_{c}^{n}|f(r, s, t)|^{2 \alpha+1}\left|D_{3} D_{2} D_{1} f(r, s, t)\right| d t d s d r \\
\leq & \frac{1}{2(\alpha+1)}(M)^{2 \alpha+1} \int_{a}^{k} \int_{b}^{m} \int_{c}^{n}\left|D_{3} D_{2} D_{1} f(r, s, t)\right|^{2(\alpha+1)} d t d s d r,
\end{aligned}
$$


which in turn, when $\alpha=0$ reduces to the inequality given in Theorem $\mathrm{B}$, where $M$ is defined by (2.3).

\section{Proof of Theorem 1}

In order to prove Theorem 1 we obtain the estimates in the following eight cases.

Case 1. Let $(r, s, t) \in \triangle_{1}$ and define

$$
h(r, s, t)=\int_{a}^{r} \int_{b}^{s} \int_{c}^{t}\left|D_{3} D_{2} D_{1} f(u, v, w)\right| d w d v d u .
$$

From (3.1) we obtain

$$
D_{1} h(r, s, t)=\int_{b}^{s} \int_{c}^{t}\left|D_{3} D_{2} D_{1} f(r, v, w)\right| d w d v,
$$

and

$$
D_{2} D_{1} h(r, s, t)=\int_{c}^{t}\left|D_{3} D_{2} D_{1} f(r, s, w)\right| d w .
$$

From the hypothesis $\left(A_{1}\right)$, it is easy to observe that

$$
\begin{gathered}
|f(r, s, t)| \leq \int_{a}^{r}\left|D_{1} f(u, s, t)\right| d u \\
\left|D_{1} f(r, s, t)\right| \leq \int_{b}^{s}\left|D_{2} D_{1} f(r, v, t)\right| d v, \\
\left|D_{2} D_{1} f(r, s, t)\right| \leq \int_{c}^{t}\left|D_{3} D_{2} D_{1} f(r, s, w)\right| d w .
\end{gathered}
$$

From (3.4)-(3.6) and (3.1)-(3.3) we observe that

$$
\begin{aligned}
|f(r, s, t)| & \leq h(r, s, t), \\
\left|D_{1} f(r, s, t)\right| & \leq D_{1} h(r, s, t), \\
\left|D_{2} D_{1} f(r, s, t)\right| & \leq D_{2} D_{1} h(r, s, t) .
\end{aligned}
$$

Using the hypotheses $\left(\mathrm{A}_{1}\right),\left(\mathrm{A}_{2}\right)$ and from (3.1) the fact that $h(r, s, t)$ is nondecreasing for $s$ and $t$ on $[b, y]$ and $[c, z]$ respectively and (3.7), (3.3), (3.2) we observe that

$$
\begin{aligned}
J_{1} & =\int_{a}^{x} \int_{b}^{y} \int_{c}^{z}\left|H(f(r, s, t)) D_{3} D_{2} D_{1} f(r, s, t)\right| d t d s d r \\
& \leq \int_{a}^{x} \int_{b}^{y} \int_{c}^{z} H(|f(r, s, t)|)\left|D_{3} D_{2} D_{1} f(r, s, t)\right| d t d s d r \\
& \leq \int_{a}^{x} \int_{b}^{y} \int_{c}^{z} H(h(r, s, t))\left|D_{3} D_{2} D_{1} f(r, s, t)\right| d t d s d r
\end{aligned}
$$




$$
\begin{aligned}
& \leq \int_{a}^{x} H(h(r, y, z)) \int_{b}^{y} \int_{c}^{z}\left|D_{3} D_{2} D_{1} f(r, s, t)\right| d t d s d r \\
& =\int_{a}^{x} H(h(r, y, z)) \int_{b}^{y} D_{2} D_{1} h(r, s, z) d s d r \\
& =\int_{a}^{x} H(h(r, y, z)) D_{1} h(r, y, z) d r \\
& =\int_{0}^{h(x, y, z)} H(\sigma) d \sigma \\
& =F(h(x, y, z)) \\
& =F\left(\int_{a}^{x} \int_{b}^{y} \int_{c}^{z}\left|D_{3} D_{2} D_{1} f(r, s, t)\right| d t d s d r\right) .
\end{aligned}
$$

Case 2. Let $(r, s, t) \in \triangle_{2}$ and define

$$
h(r, s, t)=\int_{a}^{r} \int_{b}^{s} \int_{t}^{n}\left|D_{3} D_{2} D_{1} f(u, v, w)\right| d w d v d u .
$$

Case 3. Let $(r, s, t) \in \triangle_{3}$ and define

$$
h(r, s, t)=\int_{a}^{r} \int_{s}^{m} \int_{c}^{t}\left|D_{3} D_{2} D_{1} f(u, v, w)\right| d w d v d u .
$$

Case 4. Let $(r, s, t) \in \triangle_{4}$ and define

$$
h(r, s, t)=\int_{r}^{k} \int_{b}^{s} \int_{c}^{t}\left|D_{3} D_{2} D_{1} f(u, v, w)\right| d w d v d u .
$$

Case 5. Let $(r, s, t) \in \triangle_{5}$ and define

$$
h(r, s, t)=\int_{a}^{r} \int_{s}^{m} \int_{t}^{n}\left|D_{3} D_{2} D_{1} f(u, v, w)\right| d w d v d u .
$$

Case 6. Let $(r, s, t) \in \triangle_{6}$ and define

$$
h(r, s, t)=\int_{r}^{k} \int_{s}^{m} \int_{c}^{t}\left|D_{3} D_{2} D_{1} f(u, v, w)\right| d w d v d u .
$$

Case 7. Let $(r, s, t) \in \triangle_{7}$ and define

$$
h(r, s, t)=\int_{r}^{k} \int_{b}^{s} \int_{t}^{n}\left|D_{3} D_{2} D_{1} f(u, v, w)\right| d w d v d u .
$$


Case 8. Let $(r, s, t) \in \triangle_{8}$ and define

$$
h(r, s, t)=\int_{r}^{k} \int_{s}^{m} \int_{t}^{n}\left|D_{3} D_{2} D_{1} f(u, v, w)\right| d w d v d u .
$$

Now, by following similar arguments to those in the proof of Case 1, but with suitable modifications, we obtain the following estimates in Cases 2-8:

$$
\begin{aligned}
& J_{2}=\int_{a}^{x} \int_{b}^{y} \int_{z}^{n}\left|H(f(r, s, t)) D_{3} D_{2} D_{1} f(r, s, t)\right| d t d s d r \\
& \leq F\left(\int_{a}^{x} \int_{b}^{y} \int_{z}^{n}\left|D_{3} D_{2} D_{1} f(r, s, t)\right| d t d s d r\right), \\
& J_{3}=\int_{a}^{x} \int_{y}^{m} \int_{c}^{z}\left|H(f(r, s, t)) D_{3} D_{2} D_{1} f(r, s, t)\right| d t d s d r \\
& \leq F\left(\int_{a}^{x} \int_{y}^{m} \int_{c}^{z}\left|D_{3} D_{2} D_{1} f(r, s, t)\right| d t d s d r\right), \\
& J_{4}=\int_{x}^{k} \int_{b}^{y} \int_{c}^{z}\left|H(f(r, s, t)) D_{3} D_{2} D_{1} f(r, s, t)\right| d t d s d r \\
& \leq F\left(\int_{x}^{k} \int_{b}^{y} \int_{c}^{z}\left|D_{3} D_{2} D_{1} f(r, s, t)\right| d t d s d r\right), \\
& J_{5}=\int_{a}^{x} \int_{y}^{m} \int_{z}^{n}\left|H(f(r, s, t)) D_{3} D_{2} D_{1} f(r, s, t)\right| d t d s d r \\
& \leq F\left(\int_{a}^{x} \int_{y}^{m} \int_{z}^{n}\left|D_{3} D_{2} D_{1} f(r, s, t)\right| d t d s d r\right), \\
& J_{6}=\int_{x}^{k} \int_{y}^{m} \int_{c}^{z}\left|H(f(r, s, t)) D_{3} D_{2} D_{1} f(r, s, t)\right| d t d s d r \\
& \leq F\left(\int_{x}^{k} \int_{y}^{m} \int_{c}^{z}\left|D_{3} D_{2} D_{1} f(r, s, t)\right| d t d s d r\right), \\
& J_{7}=\int_{x}^{k} \int_{b}^{y} \int_{z}^{n}\left|H(f(r, s, t)) D_{3} D_{2} D_{1} f(r, s, t)\right| d t d s d r \\
& \leq F\left(\int_{x}^{k} \int_{b}^{y} \int_{z}^{n}\left|D_{3} D_{2} D_{1} f(r, s, t)\right| d t d s d r\right), \\
& J_{8}=\int_{x}^{k} \int_{y}^{m} \int_{z}^{n}\left|H(f(r, s, t)) D_{3} D_{2} D_{1} f(r, s, t)\right| d t d s d r \\
& \leq F\left(\int_{x}^{k} \int_{y}^{m} \int_{z}^{n}\left|D_{3} D_{2} D_{1} f(r, s, t)\right| d t d s d r\right) .
\end{aligned}
$$


From (3.10), (3.18)-(3.24), it follows that

$$
\begin{aligned}
& \int_{a}^{k} \int_{b}^{m} \int_{c}^{n}\left|H(f(r, s, t)) D_{3} D_{2} D_{1} f(r, s, t)\right| d t d s d r \\
= & \sum_{j=1}^{8} J_{j} \\
\leq & \sum_{j=1}^{8} F\left(I_{j} D_{3} D_{2} D_{1} f\right) .
\end{aligned}
$$

This completes the proof.

\section{Proof of Theorem 2}

In order to prove Theorem 2, we require to obtain estimates in the Cases 1-8 as discussed in the proof of Theorem 1 . We consider the details of Case 1 as follows.

Case 1. Let $(r, s, t) \in \triangle_{1}$ and define a function $h(r, s, t)$ by (3.1) in the proof of Case 1 in Theorem 1. As in the proof of Case 1 in Theorem 1, (3.2)-(3.9) hold. Then by using the hypotheses $\left(\mathrm{A}_{1}\right),\left(\mathrm{A}_{2}\right)$ and from $(3.1)$ the fact that $h(r, s, t)$ is nondecreasing for $s$ and $t$ on $[b, y]$ and $[c, z]$ respectively and (3.7), (3.3), (3.2), we observe that

$$
\begin{aligned}
J_{1} & =\int_{a}^{x} \int_{b}^{y} \int_{c}^{z}\left|\frac{D_{3} D_{2} D_{1} f(r, s, t)}{H(f(r, s, t))}\right| d t d s d r \\
& \geq \int_{a}^{x} \int_{b}^{y} \int_{c}^{z} \frac{\left|D_{3} D_{2} D_{1} f(r, s, t)\right|}{H(|f(r, s, t)|)} d t d s d r \\
& \geq \int_{a}^{x} \int_{b}^{y} \int_{c}^{z} \frac{\left|D_{3} D_{2} D_{1} f(r, s, t)\right|}{H(h(r, s, t))} d t d s d r \\
& \geq \int_{a}^{x} \frac{1}{H(h(r, y, z))} \int_{b}^{y} \int_{c}^{z}\left|D_{3} D_{2} D_{1} f(r, s, t)\right| d t d s d r \\
& =\int_{a}^{x} \frac{1}{H(h(r, y, z))} D_{1} h(r, y, z) d r \\
& =\int_{0}^{h(x, y, z)} \frac{1}{H(\sigma)} d \sigma \\
& =G(h(x, y, z)) \\
& =G\left(\int_{a}^{x} \int_{b}^{y} \int_{c}^{z}\left|D_{3} D_{2} D_{1} f(r, s, t)\right| d t d s d r\right) .
\end{aligned}
$$

The estimates in Cases 2-8 as in the proof of Theorem 1 can be obtained by following the similar arguments as in the proof of Case 1 given above but with suitable modifications. We omit the further details of the proof. 


\section{Proof of Theorem 3}

To prove Theorem 3, we obtain estimates in the Cases 1-8 as discussed in the proof of Theorem 1. We give the details of Case 1 as follows.

Case 1. Let $(r, s, t) \in \triangle_{1}$ and define a function $h(r, s, t)$ by $(3.1)$ in the proof of Case 1 in Theorem 1. As in the proof of Case 1 in Theorem 1, (3.2)-(3.9) hold. Then by using the hypotheses $\left(\mathrm{A}_{1}\right),\left(\mathrm{A}_{3}\right)$ and from (3.1) the fact that $h(r, s, t)$ is nondecreasing for $s$ and $t$ on $[b, y]$ and $[c, z]$ respectively and (3.7), (3.3), (3.2) we observe that

$$
\begin{aligned}
J_{1} & =\int_{a}^{x} \int_{b}^{y} \int_{c}^{z} H^{\prime}(|f(r, s, t)|)\left|D_{3} D_{2} D_{1} f(r, s, t)\right| d t d s d r \\
& \leq \int_{a}^{x} \int_{b}^{y} \int_{c}^{z} H^{\prime}(h(r, s, t))\left|D_{3} D_{2} D_{1} f(r, s, t)\right| d t d s d r \\
& \leq \int_{a}^{x} H^{\prime}(h(r, y, z)) \int_{b}^{y} \int_{c}^{z}\left|D_{3} D_{2} D_{1} f(r, s, t)\right| d t d s d r \\
& =\int_{a}^{x} H^{\prime}(h(r, y, z)) D_{1} h(r, y, z) d r \\
& =\int_{a}^{x} \frac{d}{d r} H(h(r, y, z)) d r \\
& =H(h(x, y, z)) \\
& =H\left(\int_{a}^{x} \int_{b}^{y} \int_{c}^{z}\left|D_{3} D_{2} D_{1} f(r, s, t)\right| d t d s d r\right) .
\end{aligned}
$$

The estimates in Cases 2-8 can be obtained by following the similar arguments as in the proof of Case 1 and in view of the proof of Theorem 1 with suitable modifications. Here we omit the further details.

\section{Proof of Theorem 4}

To establish the inequality (2.5), we consider the following eight cases.

Case 1. Let $(r, s, t) \in \triangle_{1}$ and define a function $h(r, s, t)$ by (3.1) in the proof of Case 1 in Theorem 1. As in the proof of Case 1 in Theorem 1, (3.2)-(3.9) hold. Then by using the hypotheses $\left(\mathrm{A}_{1}\right),\left(\mathrm{A}_{4}\right)$ and by the same reasoning as in the proof of Case 1 in Theorem 1 , we observe that

$$
\begin{aligned}
J_{1} & =\int_{a}^{x} \int_{b}^{y} \int_{c}^{z} L\left[f, D_{3} D_{2} D_{1} f, H, H^{\prime}\right] d t d s d r \\
& \leq \int_{a}^{x} \int_{b}^{y} \int_{c}^{z}\left[H(h(r, s, t))+H^{\prime}(h(r, s, t)) h(r, s, t)\right]\left|D_{3} D_{2} D_{1} f(r, s, t)\right| d t d s d r \\
& \leq \int_{a}^{x}\left[H(h(r, y, z))+H^{\prime}(h(r, y, z)) h(r, y, z)\right] \int_{b}^{y} \int_{c}^{z}\left|D_{3} D_{2} D_{1} f(r, s, t)\right| d t d s d r
\end{aligned}
$$




$$
\begin{aligned}
& =\int_{a}^{x}\left[H(h(r, y, z))+H^{\prime}(h(r, y, z)) h(r, y, z)\right] D_{1} h(r, y, z) d r \\
& =\int_{a}^{x} \frac{d}{d r}[H(h(r, y, z)) h(r, y, z)] d r \\
& =H(h(x, y, z)) h(x, y, z) \\
& =H\left(I_{1} D_{3} D_{2} D_{1} f\right)\left(I_{1} D_{3} D_{2} D_{1} f\right) .
\end{aligned}
$$

The proof of the remaining seven cases follow by the same arguments as those of given in Theorem 1 and in view of the proof of Case 1 given above. Here we omit the further details.

\section{Proof of Theorem 5}

In order to establish the inequality (2.6), we obtain the estimates in the following eight cases.

Case 1. Let $(r, s, t) \in \triangle_{1}$ and for $i=1,2, \ldots, N$ define

$$
h_{i}(r, s, t)=\int_{a}^{r} \int_{b}^{s} \int_{c}^{t}\left|D_{3} D_{2} D_{1} f_{i}(u, v, w)\right| d w d v d u .
$$

From (5.1) and in view of the hypotheses $\left(\mathrm{A}_{5}\right)$ and $\left(\mathrm{A}_{6}\right)$, it is easy to observe that (3.2) to (3.9) hold by replacing $h$ and $f$ respectively by $h_{i}$ and $f_{i}$ for $i=1,2, \ldots, N$. Then by hypotheses $\left(\mathrm{A}_{5}\right),\left(\mathrm{A}_{6}\right)$ and by using the same reasoing as in the proof of Case 1 in Theorem 1 with suitable modifications we observe that

$$
\begin{aligned}
J_{1}= & \int_{a}^{x} \int_{b}^{y} \int_{c}^{z} M\left[H_{1}, \ldots, H_{N}, H_{1}^{\prime}, \ldots, H_{N}^{\prime}, D_{3} D_{2} D_{1} f_{1}, \ldots, D_{3} D_{2} D_{1} f_{N}\right] d t d s d r \\
\leq & \int_{a}^{x} \int_{b}^{y} \int_{c}^{z}\left[H_{1}\left(h_{1}(r, s, t)\right) \cdots H_{N-1}\left(h_{N-1}(r, s, t)\right) H_{N}^{\prime}\left(h_{N}(r, s, t)\right)\left|D_{3} D_{2} D_{1} f_{N}(r, s, t)\right|\right. \\
& +H_{1}\left(h_{1}(r, s, t)\right) \cdots H_{N-2}\left(h_{N-2}(r, s, t)\right) H_{N-1}^{\prime}\left(h_{N-1}(r, s, t)\right) \\
& \quad \times\left|D_{3} D_{2} D_{1} f_{N-1}(r, s, t)\right| H_{N}\left(h_{N}(r, s, t)\right) \\
& \left.+\cdots+H_{1}^{\prime}\left(h_{1}(r, s, t)\right)\left|D_{3} D_{2} D_{1} f_{1}(r, s, t)\right| H_{2}\left(h_{2}(r, s, t)\right) \cdots H_{N}\left(h_{N}(r, s, t)\right)\right] d t d s d r \\
\leq & \int_{a}^{x}\left[H_{1}\left(h_{1}(r, y, z)\right) \cdots H_{N-1}\left(h_{N-1}(r, y, z)\right) H_{N}^{\prime}\left(h_{N}(r, y, z)\right)\right. \\
& \times \int_{b}^{y} \int_{c}^{z}\left|D_{3} D_{2} D_{1} f_{N}(r, s, t)\right| d t d s \\
& +H_{1}\left(h_{1}(r, y, z)\right) \cdots H_{N-2}\left(h_{N-2}(r, y, z)\right) H_{N-1}^{\prime}\left(h_{N-1}(r, y, z)\right) H_{N}\left(h_{N}(r, y, z)\right) \\
& \times \int_{b}^{y} \int_{c}^{z}\left|D_{3} D_{2} D_{1} f_{N-1}(r, s, t)\right| d t d s \\
& +\cdots+H_{1}^{\prime}\left(h_{1}(r, y, z)\right) H_{2}\left(h_{2}(r, y, z)\right) \cdots H_{N}\left(h_{N}(r, y, z)\right)
\end{aligned}
$$




$$
\begin{aligned}
& \left.\times \int_{b}^{y} \int_{c}^{z}\left|D_{3} D_{2} D_{1} f_{1}(r, s, t)\right| d t d s\right] d r \\
\leq & \int_{a}^{x}\left[H_{1}\left(h_{1}(r, y, z)\right) \cdots H_{N-1}\left(h_{N-1}(r, y, z)\right) H_{N}^{\prime}\left(h_{N}(r, y, z)\right) D_{1} h_{N}(r, y, z)\right. \\
& +H_{1}\left(h_{1}(r, y, z)\right) \cdots H_{N-2}\left(h_{N-2}(r, y, z)\right) H_{N-1}^{\prime}\left(h_{N-1}(r, y, z)\right) H_{N}\left(h_{N}(r, y, z)\right) \\
& \times D_{1} h_{N-1}(r, y, z) \\
& \left.+\cdots+H_{1}^{\prime}\left(h_{1}(r, y, z)\right) H_{2}\left(h_{2}(r, y, z)\right) \cdots H_{N}\left(h_{N}(r, y, z)\right) D_{1} h_{1}(r, y, z)\right] d r \\
= & \int_{a}^{x} \frac{d}{d r}\left[\prod_{i=1}^{N} H_{i}\left(h_{i}(r, y, z)\right)\right] d r \\
= & \prod_{i=1}^{N} H_{i}\left(h_{i}(x, y, z)\right) \\
= & \prod_{i=1}^{N} H_{i}\left(I_{1} D_{3} D_{2} D_{1} f_{i}\right) .
\end{aligned}
$$

The estimates in the Cases 2-8 can be obtained by following the proof of Theorem 1 and the similar arguments as in the proof of Case 1 given above with suitable modifications. We omit the further details.

Remark 6. The multidimensional integral inequalities of the Opial type are established by many authors, see [1, 3, 6-12] and the references given therein. Here we note that our results are obtained by using quite elementary method and we believe that the inequalities established in this paper are of independent interest.

\section{References}

[1] R. P. Agarwal and P. Y. H. Pang, Opial Inequalities with Applications in Differential and Difference Equations, Kluer, Dordrecht, 1995.

[2] D. S. Mitrinovic, Analytic Inequalities, Springer-Verlag, Berlin, New York, 1970.

$[3]$ D. S. Mitrinovic, J. E. Peceric and A. M. Fink, Inequalities Involving Functions and their Integrals and Derivatives, Kluwer, Dordrecht, 1991.

[4] Z. Opial, Sur une inegalite, Ann. Polon. Math. 8(1960), 29-32.

[5] B. G. Pachpatte, On Opial type inequalities in two independent variables, Proc. Royal. Soc. Edinburgh 100A(1985), 263-270.

[6] B. G. Pachpatte, On Yang type integral inequalities, Tamkang J. Math. 18(1987), 89-96.

[7] B. G. Pachpatte, On two inequalities similar to Opial's inequality in two independent variables, Periodica Math. Hungarica 18(1987), 137-141.

[8] B. G. Pachpatte, On two independent variable Opial type integral inequalities, J. Math. Anal. Appl. 125(1987), 47-57.

[9] B. G. Pachpatte, On certain two dimensional integral inequalities, Chinese. Jour. Math. 17(1989), 273-279. 
[10] B. G. Pachpatte, On an integral inequality in two variables, Demonstratio Mathematica 23(1990), 593-597.

[11] Sh. Salem, On Opial type inequalities in three independent variables, Kyungpook Math. J. 36(1986), 63-72.

[12] G. S. Yang, Inequality of Opial type in two variables, Tamkang J. Math. 13(1982), 255-259.

57 Shri Niketan Colony, Near Abhinay Talkies, Aurangabad 431 001, (Maharashtra), India. 